OG $6.1-4$

338

\title{
SECONDARY ANTIPROTON PRODUCTION IN RELATIVISTIC PLASMAS
}

\author{
C. D. Dermer* and R. Ramaty \\ NASA/Goddard Space Flight Center \\ Greenbelt, MD 20771
}

I. Introduction. We investigate the possibility that the reported excess low energy antiproton component of the cosmic radiation results from proton-proton ( $p-p)$ interactions in relativistic plasmas. Because of both target and projectile motion in such plasmas, the antiproton production threshold in the frame of the plasma is much lower than the threshold of antiproton production in cosmic ray interactions with ambient matter. The spectrum of the resultant antiprotons therefore extends to much lower energy than in the cosmic ray case.

We calculate the antiproton spectrum for relativistic thermal plasmas and estimate the spectrum for relativistic nonthermal plasmas. As possible production sites, we consider matter accreting onto compact objects located in the galaxy. Possible overproduction of $\gamma$-rays from associated $\pi^{\circ}$ production can be avoided if the site is optically thick to the photons but not to the antiprotons. A possible scenario involves a sufficiently large photon density that the $\pi^{\circ} \gamma$-rays are absorbed by $\gamma-\gamma$ pair production. Escape of the antiprotons to the interstellar medium can be mediated by antineutron production.

II. Observations and Constraints on Secondary Production Models. Golden et al. (1979) have reported an antiproton to proton $(\bar{p} / \mathrm{p})$ ratio of $5.2( \pm 1.5) \times 10^{-4}$ in the cosmic radiation in the energy range 4.7-11.6 GeV. Bogomolov et al. (1979), on the basis of only two events, report a $\bar{p} / \mathrm{p}$ ratio of $6( \pm 4) \times 10^{-4}$ at energies between 2 and $5 \mathrm{GeV}$. Buffington et al. (1981) report a $\bar{p} / \mathrm{p}$ ratio of $2.2( \pm 0.6) \times 10^{-4}$ between 130 and 320 $\mathrm{MeV}$, and also determine that the antihelium to helium ratio $\bar{\alpha} / \alpha<2.2 x$ $10^{-5}$ in the energy range $130-370 \mathrm{MeV} /$ nucleon. Upper limits on prior antinucleus searches can also be found in this paper.

As is well known, the simple "leaky box" model of cosmic ray propagation predicts a $\bar{p} / p$ ratio smaller by a factor of $3-5$ than the values measured in the Golden and Bogomolov experiments. Because of the kinematic cutoff associated with the high $\bar{p}$ production threshold in $p-p$ collisions when one of the protons is at rest, the $\bar{p} / p$ ratio predicted by this model is some two orders of magnitude lower than the value reported by Buffington et al., even after the effects of solar modulation are taken into account ( $\operatorname{Tan}$ and $\mathrm{Ng} 1983 \mathrm{a}$ ). The low $\bar{\alpha} / \alpha$ ratio, in comparison with the $\bar{p} / p$ ratios, suggests a secondary origin of the $\bar{p}$, although primary cosmic ray antiproton theories, with subsequent breakdown of the $A>1$ antinuclei, have been proposed (e.g., Stecker et al. 1983).

Theories of secondary $\bar{p}$ production in $p-p$ collisions in the galaxy are constrained by the observed galactic gamma-ray luminosity. The observed antiproton flux $\Phi_{\bar{p}}(E)$ implies a total galactic $\bar{p}$ production rate

*NAS/NRC Resident Research Associate 


\section{9}

OG $6.1-4$

$$
Q(\bar{p}) \sim \frac{4 \pi V}{c} \int_{0}^{\infty} d E \frac{\Phi-(E)}{\beta \cdot \tau(E)} \sim\left(3.5 \times 10^{-14} V / \tau\right) \bar{p} / \sec ,
$$

where $V$ is the galactic confinement volume and $\tau$ is the average galactic $\overline{\mathrm{p}}$ residence time. Taking $\mathrm{V} \sim \pi(15 \mathrm{kpc})^{2}(1 \mathrm{kpc}) \sim 1.2 \times 10^{67} \mathrm{~cm}^{3}$ and $\tau \sim$ $10^{7}$ yrs gives $Q(\bar{p}) \sim 2.1 \times 10^{39} \bar{p} / \mathrm{sec}$. If the production of a secondary $\bar{p}$ is accompanied by the production of $\langle n\rangle \pi^{\circ}$-decay gamma rays, the resultant $\gamma$-ray luminosity of the galaxy $Q(\gamma) \sim\langle n\rangle Q(\bar{p})$. In the case of the production of $>100 \mathrm{MeV}$ photons in cosmic ray interactions, $\langle n\rangle_{c r} \sim$ $2.7 \times 10^{3}$, from the calculations of Stephens and Badhwar (1981) and Tan and $\mathrm{Ng}$ (1983a). If the $\overline{\mathrm{p}}$ in the cosmic radiation are entirely cosmic ray secondaries, the $>100 \mathrm{MeV}$ luminosity of the galaxy should therefore be at least $5.7 \times 10^{42} \mathrm{\gamma} / \mathrm{sec}$, in contrast to the measured value of $\sim$ $2.5 \times 10^{4+2} \mathrm{r} / \mathrm{sec}$ from the work of Bloemen et al. (1984). This latter number is an upper limit, since it includes a significant contribution from bremsstrahlung and inverse Compton $\gamma$-rays.

Various models have been designed to either increase the $\bar{p}$ lifetime $\tau$, incorporate additional $\bar{p}$ sources, or conceal the $\pi^{\circ} \gamma$-rays. Although the integral production rate of $\bar{p}$ can then be made to agree with observations, most models still fail to predict a substantial low energy $\vec{p}$

flux because of the low energy cutoff that results from secondary interactions with stationary targets, in disagreement with the experiment of Buffington et al. (1981). Models that provide deceleration of the $\bar{p}$

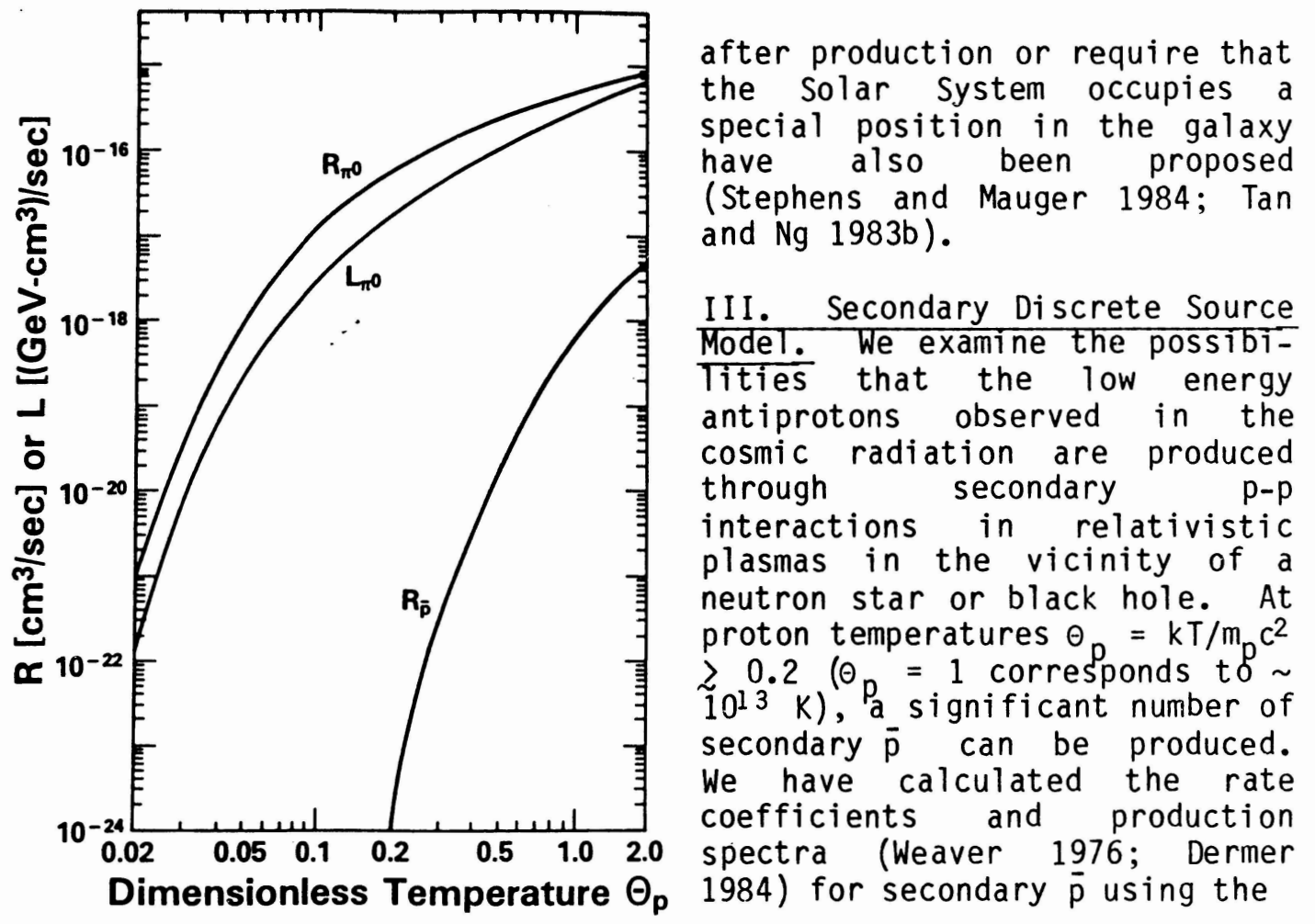

Fig. 1. The rate coefficients for secondary $\pi^{\circ}$ and $\bar{p}$ production from $p-$ $p$ collisions in a relativistic plasma at temperature $\theta_{0}=\mathrm{kT} / \mathrm{m} c^{2}$. Al so shown is the $\pi^{\circ}$ luminosity coefficient giving the Potal ehergy in secondary $\pi^{\circ} \gamma$-rays produced at temperature $\theta_{p}$. 


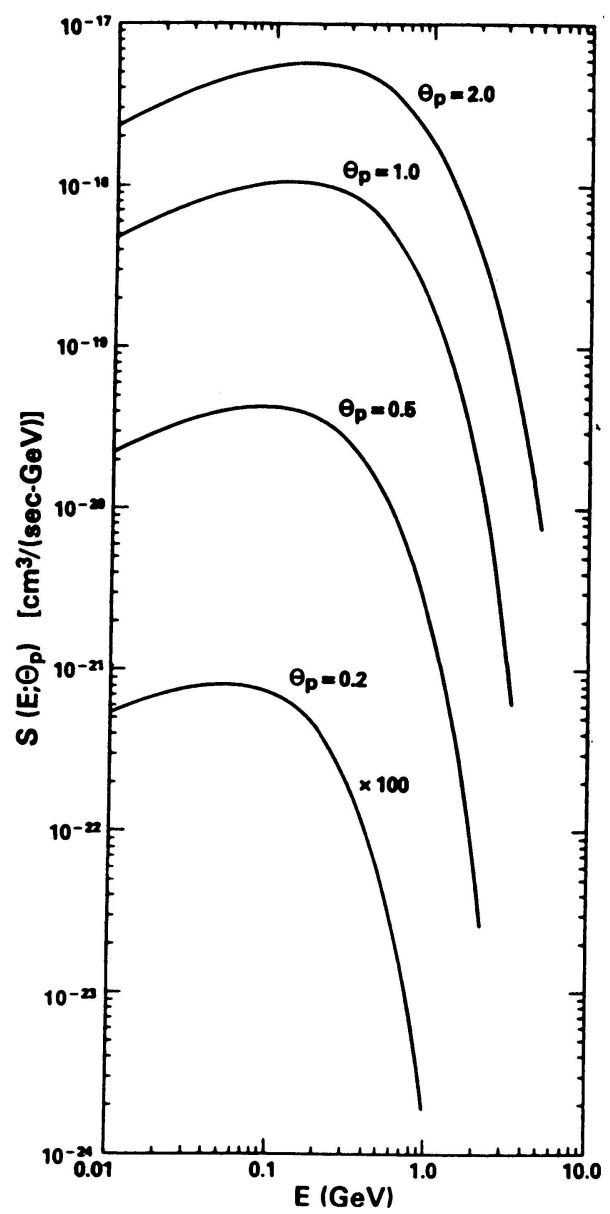

invariant cross section of Tan and $\mathrm{Ng}$ (1982) and the results are shown in Figs. 1 and 2. We have also calculated the rate and luminosity coefficients from secondary $\pi^{\circ}$ production data. From Fig. 1 we find that the efficiency for $\bar{p}$ production compared to $\pi^{\circ}$ production is greater than in the cosmic ray case for $\theta_{p} \geqslant 0.5$, so the total $\pi^{\circ}$-decay $\gamma$-ray pluminosity will not exceed limits implied by $\gamma$-ray observations of the galaxy. In addition, a number of absorption processes likely to occur near compact objects, such as photon-photon or magnetic pair production, can further reduce the $\pi^{\circ} \gamma$-ray luminosity.

The $\bar{p}$ spectra shown in Fig.2 for a variety of temperatures extend to low energies without the appearance of the kinematic cutoff found in the cosmic ray problem. The spectra peak at higher energies with increasing temperature, and exhibit an exponential decline above the peak temperature. But in all cases a very low energy secondary $\bar{p}$ component is calculated, which could possibly explain the low energy $\bar{p}$ observation of Buffington et al. (1981).

Fig. 2. Secondary $\bar{p}$ production spectra at various temperatures $\theta_{p}=k T / m_{p} c^{2}$ are given as a function of kinetic energy $E$.

IV. Discussion. Possible production sites of the $\bar{p}$ 's include the galactic bulge binary $X-r$ ay sources or the objects associated with the $\gamma$-ray point sources (e.g. Cyg $X-3$ ). Models for disk accretion (Eilek 1980) and spherical accretion incorporating shocks (Meszaros and Ostriker 1983) could yield proton temperatures as high as $\theta_{p} \sim 1$. Although the establishment of a thermal distribution of protons may not be possible at these temperatures, this assumption affords the simplest calculations. Preliminary estimates employing nonthermal proton spectra in relativistic plasmas suggest low energy $\bar{p}$ spectra similar in form to the results of Fig. 2 .

Transport of the secondary $\bar{p}$ from the production site can occur because antineutrons $\bar{n}$, produced in equal numbers as the $\bar{p}$ (Gaisser and Maurer 1973), are not confined by the ambient plasma magnetic field. The $\bar{n}$ will escape to the interstellar medium and subsequently decay into $\bar{p}$ (the calculations of Figs. 1 and 2 refer only to these particles). Because of the finite lifetime of the $\bar{n}$, an upper limit can be placed on the mass of black holes that serve as production sites for the $\bar{p}$. Requiring that the marginally relativistic $\bar{n}$ escape to 10 Schwarzschild 


\section{1}

radii implies a maximum black hole mass of $<107 \mathrm{M}_{0}$. Past activity around such a massive black hole at the Galactic Center could have produced the antiprotons without a large contemporaneous gamma emission. A detailed treatment of $\bar{p}$ production in a relativistic plasma must also include distortions of the emergent $\bar{n}$ and $\gamma-r$ ay spectrum due to the intense gravitational field of the compact object, and possible reacceleration of the source spectrum in the galactic environment.

v. Conclusions. We have presented a model that can produce low energy antiprotons through secondary $p-p$ interactions in relativistic plasmas. Such a model is in agreement with the observation of low energy antiprotons in the cosmic radiation and the observed $\gamma$-ray luminosity of the galaxy. Moreover, it agrees with the present lack of observations of antinuclei in the cosmic radiation, whose formation by secondary production processes is entirely negligible.

\section{References}

J. B. G. M. Bloemen et al. 1984, Ap. J., 279, 136.

E. A. Bogomolov et a1. 1979, Proc. 16th I ICRC (Kyoto), 1, 330.

A. Buffington et al. 1981, Ap. J., 248, 1179.

C. D. Dermer 1984, Ap. J., 280, 328.

J. A. Eilek 1980, Ap. J., 236,664 .

T. K. Gaisser and R.H. Maurer 1973, Phys. Rev. Letters, 30, 1264.

R. L. Golden et al. 1979, Phys. Rev. Letters, 43, 1196.

P. Meszaros and J.P. Ostriker 1983, Ap. J., 273, L59.

F. W. Stecker et a1., 1983, Astrophy. Space Sci. , 96, 171.

S. A. Stephens and G.D. Badhwar, Astrophy. Space Sci., 76, 213.

S. A. Stephens and B. G. Mauger, in "High Energy Astrophysics", proceedings of the Nineteenth Recontre de Moriond Astrophysics Meeting, p. 217.

L. C. Tan and L. K. Ng 1982, Phys. Rev. D., 26, 1179.

L. C. Tan and L. K. Ng 1983a, J. Phys. G, 9, 227.

L. C. Tan and L. K. Ng 1983b, Ap. J., 269, 751 .

T. A. Weaver 1976, Phys. Rev. A., 13, 1563 . 